\title{
Monte Carlo Simulation of Interacting Polymer Systems I. Behavior of Ring Chain Interacting via Soft-Core Potential
}

\author{
Takao MinATo and Akira HATANO* \\ Nippon Information Industry Co., Ltd., \\ Dai-hyaku Seimei Shibuya Bldg., \\ Shibuya-ku, Tokyo 150, Japan \\ * Department of Pure and Applied Sciences, \\ College of General Education, The University of Tokyo, \\ 3-8-1, Komaba, Meguro-ku, Tokyo 153, Japan
}

(Received March 25, 1982)

\begin{abstract}
The excluded-volume effects on polymers were studied by a method developed for a random walk problem by Tsuda et al. on the basis of Feynman's path integral concept. The method was applied to ring chains interacting through a soft-core potential, i.e., $V(r)=\gamma$ for $r<d$ and $V(r)=0$ for $r \geqq d$. The exponent $v$ which characterizes the dependence of the chain dimensions on $N$ (number of segments) was found to be about 0.58 for repulsive interaction. However, no definite value of $v$ was obtained for attractive interaction because of large statistical scatters. An investigation was also made for chain dimensions as a function of $N^{1 / 2}, \gamma$, and $N^{1 / 2} \gamma$.

KEY WORDS Monte Carlo / Path Integral / Square Radius of Gyration / Soft-Core Potential / Ring Chain /
\end{abstract}

The purpose of the present paper is to apply the well-known method of numerical computation developed by Tsuda, Ichida and Kiyono ${ }^{1}$ to the analysis of the statistical properties of interacting polymer systems, and to present the preliminary results obtained. This method was originally devised for finding an initial solution to a non-linear diffusion equation which could not be solved analytically. We show that this method can be applied as well to the polymer problems in consideration of the similarity between the diffusion process and the random walk model of polymer chains. Our task has been to evaluate approximately the average of observable quantities by the Monte Carlo simulation on the basis of Feynman's path integral concept. ${ }^{2}$

The object of numerical experiments on polymers has so far been limited primarily the single-chain, with a flexible chain in a dilute solution being conceived as a self-avoiding random walk on a simple lattice. Two methods were established. One was the exact counting of self-avoiding walks of some very short chains, supplemented by certain techniques for extrapolating the results of very long chains. ${ }^{3}$ Another was the Monte Carlo method in which only a small fraction of all possible conformations are generated and used as samples. ${ }^{4}$ Since these numerical methods allow polymer statistics to be studied in the frame of a simple lattice model, they are extremely usefull and play an important role in evaluating various exponents characterizing the chain dimensions and their distributions. The most important exponent is concerned with the asymptotic behavior of the chain dimensions for large values of $N$ (number of segments). As is well known, the mean-square radius of gyration follows the power law $\left\langle S^{2}\right\rangle \simeq N^{2 v}$. Either of the above-mentioned numerical methods yields $v \simeq 0.6$, which is in good agreement with the most reliable theoretical prediction by the renormalization group theory. ${ }^{5}$ Recent progress in the Monte Carlo method owes much to Baumgärtner et al. ${ }^{6}$ and Lebowitz et al. ${ }^{7}$ who extensively studied polymer systems interacting via the Lennard-Jones $12-6$ po- 
tential, using models which are free from constraint of the lattice. Such a system is certainly much closer to real polymer systems than any lattice chain. Using the dynamic Monte Carlo method, these authors carefully studied complex static and dynamic behavior of a polymer chain to which the usual static Monte Carlo method cannot be applied. The results obtained showed for the first time that the exponent $v$ is about 0.59 for the Lennard-Jones 12-6 potential in which the repulsive force is dominant, and gave good insight into the dependence of condensation phenomena on temperature, chain response to external forces, and such timedependent phenomena as the diffusion or relaxation process of the chain.

It may be considered that the static or dynamic Monte Carlo method can be used to study not only single-chain problems but also the properties of many-chain systems. ${ }^{8}$ Recent scaling approaches to semi-dilute and confined polymer solutions seem to be very appealing. ${ }^{9}$ It is, therefore, of great interest to study carefully information obtained by these approaches from Monte Carlo simulations. Of course, it is possible to extend the usual Monte Carlo method to the analysis of many-chain systems, but enormouse execution time and core memories seem necessary for performing simulations by computer. Our use of the method developed by Tsuda $e t$ al. was prompted from this consideration. This method does not allow us to deal with the dynamics of polymer systems, but it has the following advantages:

1) Recent micro-computers are capable of carrying through such simulations. The classical Monte Carlo method requires very complex algorithms ${ }^{10}$ to generate self-avoiding walks on a lattice. Therefore, an enormously long execution time and large core memories are needed when it is used for simulation of many-chain systems. On the contrary, the present method described in the following section needs only a very small number of core memories and much less execution time, because it is essentially based on a simple random walk (Wiener path) generated by the use of a recursion relation.

2) This method seems capable of being generalized for application to more complex polymer systems such as branched chains, stiff-chains, copolymers, and many-chain systems in which polymer chains interact with each other via an arbitrary potential. In doing so, some modifications suggested in the following sections should be made.

3) By using the present method, we can also handle polymer chains between reflecting barriers. Recently much attention has been focused on the statistical properties of polymers interacting with walls. ${ }^{11}$ The influence of these reflecting walls can be taken into consideration since the Wiener path is allowed to reflect at the surface to a mirror image point when it crosses the surface. ${ }^{12}$

4) We can further take into account the constraints imposed on the chain ends; thus a linear chain, a ring chain, and a chain with fixed ends can be treated.

Although the present method has these advantages, its validity as well as its extensibility must be examined carefully. For this, we investigated the excluded-volume effects of a simple ring chain with $N$ segments $(N \leqq 70)$ interacting with each other via a soft-core potential. Our results were consistent with those hitherto obtained, i.e., the exponent $v$ was about 0.58 for a repulsive interaction and the mean-square radius of gyration decreased smoothly with increasing attraction. A remarkable revelation was that the mean-square radius of gyration appeared to approach a constant for attractive interactions; it decreased to about $40-50$ percent of the value for the non-interacting case.

We also derived an expression that could be used for computer simulation of an observable through application of Feynman's path integral. The method of constructing a Wiener path is also discussed in this paper on the basis of the technique devleoped by Tsuda et al.

\section{THEORETICAL BACKGROUND}

We treat a flexible chain using a random walk model, which, for simplicity, is assumed in this chapter to be constrained in one dimension. In this model, the chain consists of a sequence of $N$ links. The probability that each link starts from $x_{i}$ and ends up at $x_{i+1}$ is given by

$$
p\left(x_{i}, x_{i+1}: \varepsilon\right)=(2 \pi \varepsilon)^{-1 / 2} \exp \left[-\left(x_{i+1}-x_{i}\right)^{2} / 2 \varepsilon\right]
$$

where $\varepsilon$ is the average square length of a link and $i$ runs from 0 to $N-1$. The entire distribution function is represented as ${ }^{13}$ 


$$
Z_{0}\left(x_{N}: N\right)=\int_{-\infty}^{\infty} \cdots \int_{-\infty}^{\infty} \prod_{i=1}^{i=N-1} \mathrm{~d} x_{i} p\left(x_{0}, x_{1}: \varepsilon\right) p\left(x_{1}, x_{2}: \varepsilon\right) \cdots p\left(x_{N-1}, x_{N}: \varepsilon\right)
$$

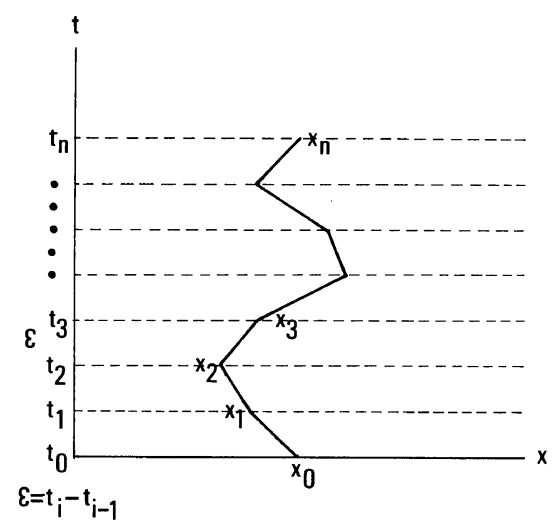

Figure 1. Representation of a Wiener path of finite step length $\varepsilon$.

where the subscript 0 to $Z_{0}$ denotes the free random walk, i.e., the unperturbed state in the polymer problem. The representation shown in Figure 1 may facilitate an understanding eq 2 . The chain is represented by a polygonal line joining $x_{0}$ to $x_{N}$ through a series of intermediate steps $x_{1}, x_{2}, \cdots, x_{N-1}$. This polygonal line may be called a "path." A path constructed in this way is a Wiener path. The entire distribution function is furnished by the assemblage of all such possible paths, and given by the integration over the intermediate positions $x_{1}, x_{2}$, $\cdots, x_{N-1}$ of the path. The fixed points $x_{0}$ and $x_{N}$ must be excluded from the integration.

Now we consider an average over all the paths $\left(x_{0}, x_{1}, \cdots, x_{N}\right)$ of an arbitrary functional $A\left(x_{0}, x_{1}\right.$, $\left.\cdots, x_{N}\right)$, which depends on the path $\left(x_{0}, x_{1}, \cdots, x_{N}\right)$ and is assumed to have a finite value for a given path:

$$
\left\langle A\left(x_{0}, x_{1}, \cdots, x_{N}\right)\right\rangle_{0}=W^{-1} \sum_{\text {all paths }} A\left(x_{0}, x_{1}, \cdots, x_{N}\right)
$$

where $W$ is the total number of paths. Since the path $\left(x_{0}, x_{1}, \cdots, x_{N}\right)$ has a probability $p\left(x_{0}, x_{1}: \varepsilon\right) p\left(x_{1}\right.$, $\left.x_{2}: \varepsilon\right) \cdots p\left(x_{N-1}, x_{N}: \varepsilon\right)$ for the free random walk, eq 3 is rewritten more explicitly as

$$
\begin{aligned}
\langle A\rangle_{0}= & Z_{0}\left(x_{N}: N\right)^{-1} \int_{-\infty}^{\infty} \cdots \int_{-\infty}^{\infty} A\left(x_{0}, x_{1}, \cdots, x_{N}\right) \\
& \times p\left(x_{0}, x_{1}: \varepsilon\right) p\left(x_{1}, x_{2}: \varepsilon\right) \cdots \\
& \times p\left(x_{N-1}, x_{N}: \varepsilon\right) \mathrm{d} x_{1} \mathrm{~d} x_{2} \cdots \mathrm{d} x_{N-1}
\end{aligned}
$$

If eq 3 were used literally, the sum could not be taken over the complete set of all possible paths but would be limited to some finite sets of paths. In order to avoid a situation of this sort, we use the path integral representation and consider the limits, $\varepsilon \rightarrow 0$ and $N \rightarrow \infty$, with $N \varepsilon=$ finite. The concept of path integral and its close connection with polymer statistics have been reviewed in detail by Freed. ${ }^{14}$ We, therefore, omit explaining all the minor details. According to this representation, eq 2 and 4 are expressed as,

$$
\begin{gathered}
Z_{0}\left(x_{N}: N\right)=\int \mathscr{D}[x(t)] \Phi\{x(t)\} \\
\langle A\rangle_{0}=Z_{0}\left(x_{N}: N\right)^{-1} \int \mathscr{D}[x(t)] A\{x(t)\} \Phi\{x(t)\}
\end{gathered}
$$

where the integral is taken over all paths satisfying $x(0)=x_{0}$ and $x(N \varepsilon)=x_{N}$, and $\Phi\{x(t)\}$ is a functional defined by

$\Phi\{x(t)\} \equiv \exp \left(-S_{0}\right)=\exp \left\{-(1 / 2) \int_{0}^{N \varepsilon}(\mathrm{d} x(t) / \mathrm{d} t)^{2} \mathrm{~d} t\right\}$

When there are intractions represented by

$$
V\left(x_{i}-x_{j}\right)
$$

between all pairs of links, eq 5 and 6 may be generalized so as to include this potential, and eq 7 is modified to

$$
\begin{array}{r}
\tilde{\Phi}\{x(t)\} \equiv \exp (-S) \equiv \exp \left(-S_{0}-S^{\prime}\right) \\
=\exp \left\{-(1 / 2) \int_{0}^{N \varepsilon}(d x(t) / \mathrm{d} t)^{2} \mathrm{~d} t\right. \\
\left.\quad-\iint_{0}^{N_{\varepsilon}} V[x(t)-x(s)] \mathrm{d} t \mathrm{~d} s\right\}
\end{array}
$$

In this case, the average of the path-dependent functional $F\{x(t)\}$ with respect to $\tilde{\Phi}\{x(t)\}$ is given by

$$
\langle F\{x(t)\}\rangle=\int \mathscr{D}[x(t)] F\{x(t)\} \tilde{\Phi}\{x(t)\} / \int \mathscr{D}[x(t)] \tilde{\Phi}\{x(t)\}
$$

Then if $A=F \exp \left(-S^{\prime}\right)$ or $A=\exp \left(-S^{\prime}\right)$ is substituted in eq 6 , it is found from eq 5 and 10 that 


$$
\begin{aligned}
& \langle F\{x(t)\}\rangle \\
= & \left\langle F\{x(t)\} \exp \left(-S^{\prime}\right)\right\rangle_{0} /\left\langle\exp \left(-S^{\prime}\right)\right\rangle_{0}
\end{aligned}
$$

The average on the r.h.s of eq 11 is taken over all paths with $x(0)=x_{0}$ and $x(N \varepsilon)=x_{N}$, and $S^{\prime}$ stands for the effects of intra-chain interactions defined by eq 7 and 9 . As already stated, the average of $F$ may be evaluated approximately by eq 3 if $F \exp \left(-S^{\prime}\right)$ and $\exp \left(-S^{\prime}\right)$ can be calculated for a given Wiener path. The presence of $\exp \left(-S^{\prime}\right)$ in both denominator and numerator allows another interpretation of the average in eq 11: eq 11 represents the weighted average of $F$ over an infinite set of Wiener paths, in which each Wiener path does not have an equal probability but has a path-dependent weight $\exp \left(-S^{\prime}\right)$.

In the descrete coordinate representation, eq 11 may be written ${ }^{14}$

$$
\begin{aligned}
& \langle F\{x(t)\}\rangle \sum_{\alpha} F\left(x_{0 \alpha}, X_{1 \alpha}, \cdots, x_{N \alpha}\right) \\
& \quad \times \exp \left(-S^{\prime}\right) / \sum_{\alpha} \exp \left(-S^{\prime}\right) \\
& S^{\prime}=\left(\beta / a^{2}\right) \varepsilon^{2} \sum_{i} \sum_{j} V\left(x_{i \alpha}-x_{j \alpha}\right) ; \quad \beta^{-1}=k T
\end{aligned}
$$

where $a$ is the length of a link, $T$ is the absolute temperature, and the subscript $\alpha$ represents $x_{\mathrm{i}}$ which is associated with the $\alpha$-th path. As already noted, the sum over $\alpha$ is inevitably restricted to the finite fraction of all possible Wiener paths in a real simulation. We adopt eq 12 as the basis for our simulation. Our task is then to generate zig-zag Wiener paths as many as possible by some appropriate method and calculate the weighted average of an observable $F$ for a given potential.

\section{EXPERIMENTAL}

\section{Wiener Path}

The method for constructing a zig-zag Wiener path (Brownian path) was presented long ago by Levy, ${ }^{15}$ and by Paley and Wiener ${ }^{16}$ (recent progress was reviewed by $\mathrm{Hida}^{17}$ ). The methods by these authors are quite general and mathematically rigorous; but not convenient for computer use. Hence, based on the method of Tsuda et al.; we employed a somewhat different and more elementary way to construct a zig-zag Wiener path as

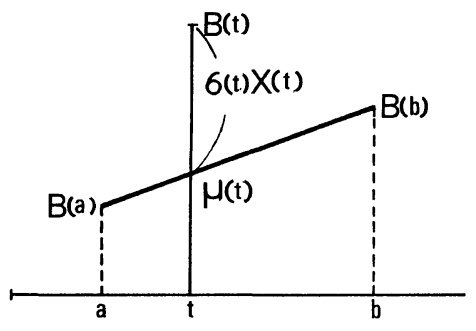

Figure 2. Graphical representation of eq 14 .

shown in Figure 1. Our method utilizes the most fundamental properties of the Brownian motion and is based also on Levy's idea.

We denote a Brownian path by $B(t)$ for $t \geqq 0$. Then if $B(a)$ and $B(b)(a<b)$ are known, the value of $B(t)$ at an arbitrary time $t$ between $a$ and $b$ is given by

$$
B(t)=\mu(t)+\sigma(t) X(t)
$$

where

$$
\begin{gathered}
\mu(t)=\{(b-t) B(a)+(t-a) B(b)\} /(b-a) \\
\sigma^{2}(t)=(t-a)(b-t) /(b-a) \quad a<t<b
\end{gathered}
$$

Here $\sigma^{2}(t)$ represents the variance of $[B(t)-\mu(t)]$ and $X(t)$ is a gaussian random variable having unit variance around the zero mean:

$$
\langle X(t)\rangle=0 ; \quad\left\langle X^{2}(t)\right\rangle=1
$$

The meaning of eq 14-16 is clear from Figure 2; i.e., $B(t)$ is the sum of the random variables $X(t) \sigma(t)$ and $\mu(t)$, the latter being the point on the straight line connecting $B(a)$ and $B(b)$. The degree of zig-zag depends on the gaussian random variable generated. Fquations $14-17$ give the general method for dividing a straight line into two parts and making a once-broken line. We can obtain the zigzag Wiener path shown in Figure 1 by repeating this dividing process at adjacent points.

Note that eq 14 can be transformed into a recursion relation convenient for computer simulation. Replacing $B(a), B(b), B(t), a, b, t$, and $X(t)$ by $x_{i-1}, x_{N}, x_{i}, t_{i-1}, t_{N}, t_{i}$, and $\xi$, respectively, we get

$$
\begin{aligned}
x_{i}= & \left\{x_{i-1}\left(t_{N}-t_{i}\right)\right. \\
& \left.x_{N}\left(t_{i}-t_{i-1}\right)\right\} /\left(t_{N}-t_{i-1}\right)+\xi \sigma \\
& \sigma^{2}=\left(t_{i}-t_{i-1}\right)\left(t_{N}-t_{i}\right) /\left(t_{N}-t_{i-1}\right)
\end{aligned}
$$

where $x_{i}$ denotes $x_{i}\left(t_{i}\right)$ and $\xi$ is a random variable obeying 


$$
\langle\xi\rangle=0 ; \quad\left\langle\xi^{2}\right\rangle=1
$$

When values are explicitly assigned to $x_{0}$ and $x_{N}$, a Wiener path $\left(x_{1}, x_{2}, \cdots, x_{N-1}\right)$ is determined through an iterative use of eq 18 . In order to describe a random walk in three dimensions, other orthogonal components $y_{i}$ and $z_{i}$ also have to be considered. These components are again determined by the use of eq 18. The relevant equations for threedimensional cases may be symbolically written in vector notations as

$$
\begin{aligned}
\vec{r}_{i}= & \left\{\vec{r}_{i-1}\left(t_{N}-t_{i}\right)\right. \\
& \left.+\vec{r}_{N}\left(t_{i}-t_{i-1}\right)\right\} /\left(t_{N}-t_{i-1}\right)+\vec{\xi} \sigma \\
\vec{r}_{i}= & \left(x_{i}, y_{i}, z_{i}\right) \quad \vec{\xi}=\left(\xi_{1}, \xi_{2}, \xi_{3}\right)
\end{aligned}
$$

where $\sigma$ is given by eq 19 and each component of $\vec{\xi}$ satisfies eq 20. Equation 21 is the basic prescription for the generation of a zig-zag Wiener path in the present experiment.

\section{Observable}

Equation 21 describes the random walk which starts from $\vec{r}_{0}$ and ends up at $\vec{r}_{N}$. Such a model, however, does not necessarily correspond to an actual polymer chain. Therefore, we put $\vec{r}_{0}=\vec{r}_{N}$ without further loss of reality. The result represents the random walk which returns to the starting point after $N$ steps of walk, and corresponds to the ring chain in polymer problems. As the observable quantity, we take the square radius of gyration defined by

$$
S^{2}=\left(2 N^{2}\right)^{-1} \sum\left(\vec{r}_{i}-\vec{r}_{j}\right)^{2}
$$

We can also examine other observables such as a local segment density or $r_{N / 2}^{2}$.

\section{Potential}

It is difficult mathematically to prove the existence of the path integral (eq 10) for a given potential. In a real simulation, we must use a potential $V(r)$ that yields the convergent sum in eq 13. Thus, the potential $V(r)$ diverging to $-\infty$ for small values of $r$ should be excluded. For this reason, we chose the following very simple potential:

$$
V(r)= \begin{cases}\gamma & \text { for } \quad r<d \\ 0 & \text { for } \quad r \geqq d\end{cases}
$$

The behavior of a freely jointed, off-lattice polymer chain interacting via this type of potential $(\gamma \rightarrow \infty)$ was studied by Baumgärtner and Binder. ${ }^{18}$

We introduce a new variable $\kappa$, which is the coefficient of $V(r)$ in eq 13 and hence depends on $a$, $\gamma, \beta$, and $\varepsilon$ as

$$
\kappa=\beta \gamma \varepsilon^{2} / a^{2}
$$

The range $d$ and the strength $\kappa$ are independent parameters which characterize the effect of the potential $V(r)$. In order to study the behavior of a chain having an attractive potential, we also consider the case of negative $\kappa$. Although pure attractive potential having no repulsive core has no physical meaning, it is by no means trivial.

\section{Parameters}

The independent parameters characterizing the polymer chain with intra-chain interactions are $\gamma, \varepsilon$, and $d$, and certain values must be assigned to these to carry out the computation. The main difficulty in the present method is that the basic unit of length such as lattice spacing does not exist for small but finite $\varepsilon$. That is, the real length of each link is not equal to a fixed $\varepsilon$ but is a random variable as seen from eq 21 . For such a random variable, only the mean square length is physically meaningful. This mean square length of a link is equal to $a^{2}$ in eq 25 and therefore is related to $\varepsilon$ through $a^{2} \simeq 3 \varepsilon$ in three dimensional cases. The distribution of link lengths gives rise to another difficulty in the calculation of the interaction energy in eq 13. There is the possibility that the nearest neighbor interactions, which should be definitely distinguished from the long range interactions, gives non-negligible contributions to the statistical average in eq 12. This unfavorable property may become more appreciable for negative values of $\kappa$ or short step walks. Keeping in mind what has been mentioned so far, and that $a \simeq d$, we use the following values for the parameters $d, \varepsilon$, and $\kappa$ :

$$
d=0.2 ; \quad \varepsilon=0.1 ; \quad-1.0 \leqq \kappa \leqq 1.2
$$

The number of samples generated was about 8000 for all $N$, and the number of steps used were 25,30 , $40,50,60$, and 70 . Tsuda et al. ${ }^{1}$ obtained, for the case in which there is a one-body potential and $N=20$ (very short steps), a good estimation for the local variable $r_{i}$ (see eq 21) from the simulation of 800 samples. We maintain, however, that more than ten thousand samples are needed for a precise estimation of the exponent $v$. 
The gaussian random number $\xi$ obtained in our experiment, by a random number $\eta_{i}$ distributed uniformly in the interval $[0,1]$ through the relation

$$
\xi=\sum_{i=1}^{12} \eta_{i}-6
$$

After having generated about 2000 samples, we changed the uniform random number series to another in order to avoid the possible periodicity of resulting random numbers.

\section{RESULTS AND DISCUSSION}

In the present experiment, the length of a link was not a constant but a random variable. Thus, the length of each path generated was different. Our statistical average of the observable $F$ in eq 12 was taken over an ensemble of such paths. The square radius of gyration defined by eq 23 was used as $F$ in our investigation. For unrestricted random walks, the average square radius of gyration is given by

$$
\left\langle S^{2}\right\rangle_{0}=N a^{2} / 12
$$

Thus, the mean-square length of a link, $a^{2}$, can be computed from the observed $\left\langle S^{2}\right\rangle_{0}$ and the chosen $N$ by means of eq 27 . The result $a^{2} \simeq 0.3$ estimated in this way agrees with the expectation and $a^{2} \simeq 3 \varepsilon$ is valid in the three dimensional case.

We first discuss the asymptotic behavior of $\left\langle S^{2}\right\rangle$ as a function of $N$, assuming that $\left\langle S^{2}\right\rangle$ follows the power law $\left\langle S^{2}\right\rangle \propto N^{2 v}$. The results are depicted in Figure 3 and tabulated in Table I. The exponent $v$ is a gradually increasing function of $\kappa$ and seems to approach 0.58 which is somewhat smaller than values obtained in other experiments ${ }^{19,21}$, and by theoretical prediction. Using the self-consistent-field approximation, Edwards first evaluated analytically the path integral of eq 10 for the delta-function type potential $(\kappa \rightarrow \infty, d \rightarrow 0$, and $\kappa d=$ finite in the present model) and showed $v=0.6$ for very large $N .^{22}$ In addition, according to the universality concept that the exponent $v$ is insensitive to the specific shape of the interaction potential, the soft-core potential is also expected to give a value near 0.6 for $v$. However, in consideration of the fact that the exact value of $v$ has not yet been established and des Cloizeaux predicted a value of $v$ somewhat smaller than $0.6,{ }^{23}$ the present result for $v$ may be regraded as reasonable. It is uncertain whether the asymptotic behavior, can be seen for small $N$ of less than

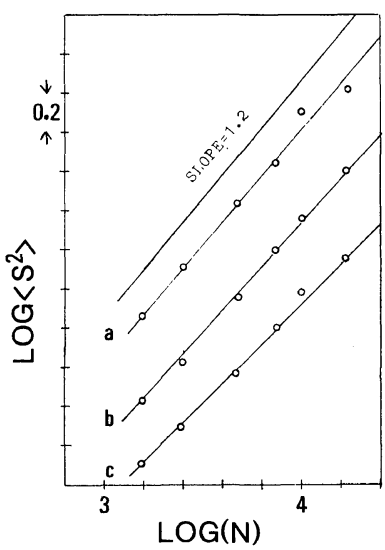

Figure 3. Log-log plot of $\left\langle S^{2}\right\rangle$ vs. $N$ : a, $\kappa=1.2$; b, $\kappa=0.6 ; c, \kappa=0$.

Table I. Exponent $v$ as a function of $\kappa$

\begin{tabular}{cc}
$\kappa$ & $2 v^{\mathrm{a}}$ \\
\hline 1.2 & 1.16 \\
1.0 & 1.15 \\
0.8 & 1.12 \\
0.6 & 1.09 \\
0.4 & 1.07 \\
0.2 & 1.03 \\
0 & 0.99 \\
-0.1 & $(0.97)^{\mathrm{b}}$ \\
-0.2 & $(0.82)$ \\
-0.3 & $(0.76)$ \\
-0.6 & $(0.82)$ \\
-1.0 & $(0.79)$ \\
\hline
\end{tabular}

a $v$ is determined by the method of least squares.

b Value in parentheses is unreliable because of large statistical errors.

70. In order to clarify this point, it is necessary to extend the computation to larger $N$ and smaller $d$. In the case of negative $\kappa$, the sum of eq 12 converges so slowly and the variance of data is so great that no definite value of $v$ could be determined. Exact enumerations based on the Monte Carlo methods in which an attractive force is introduced into selfavoiding walks, have predicted that $\left\langle S^{2}\right\rangle$ still has the asymptotic form $\left\langle S^{2}\right\rangle \propto N^{2 v}$, and that $v$ is a function of the strength of the attractive force and varies continuously from 0.6 to 0.33 . In the presence of a strong attractive force, the experiments based on the ensembles of self-avoiding walks may give $v$ values no lower than 0.33 . Although $v \simeq 0.33$ was 
Monte Carlo Simulation of Interacting Polymer Systems I.

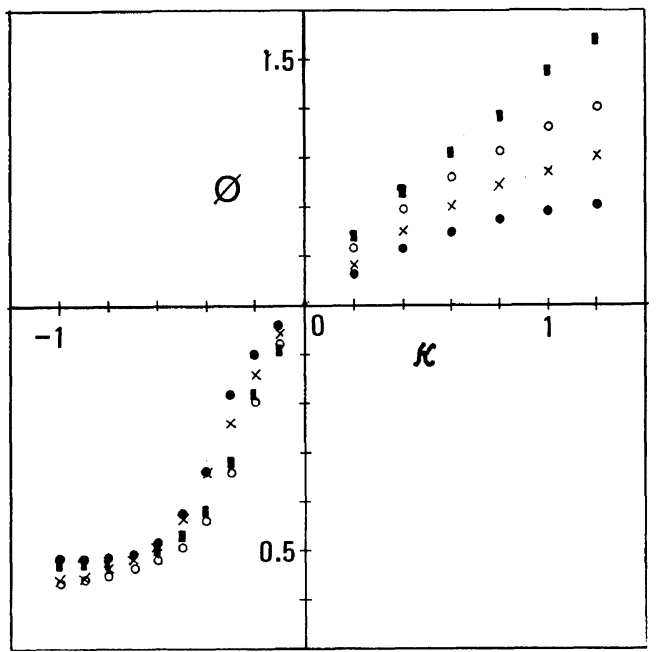

Figure 4. Expansion factor $\Phi$ as a function of $\kappa$ for various values of $N$ (number of steps): a ( $\square$ ), $N=70$; b (O), $N=60 ;$ c (×), $N=40 ; \mathrm{d}(\odot), N=25$.

obtained in our previous work, ${ }^{24}$ it is not entirely clear whether this value can be derived from an ensemble of unrestricted random walks modified by the pseudo-potential used in the present calculation.

The behavior of the renormalized mean square radius of gyration $\Phi=\left\langle S^{2}\right\rangle /\left\langle S^{2}\right\rangle_{0}$ is shown in Figure 4 as a function of $\kappa$. For negative $\kappa$, a smooth decrease in $\Phi$ occurs with decreasing $\kappa$. This tendency is intensified with increasing $N{ }^{25}$ Figure 4 shows that $\Phi$ is not a rapidly decreasing function of $\kappa$, but appears to approach a constant value, implying that even in the presence of the attractive force, the perturbed square radius of gyration has a finite expansion with a non-zero radius. As is seen from Figure 4 , the square radius of gyration decreases with decreasing $\kappa$ to about $40-50$ percent of that for the unperturbed state. It is quite correct that the expansion factor $\Phi$ vanishes when $N$ tends to infinity and remains finite when $N$ is finite. This does not mean that, for the pseudo-potential model, the chain collapses very rapidly to zero for all $N$ when $\kappa$ is negative. ${ }^{26}$ In the case of pure attraction with no repulsive core, it is necessary to investigate carefully whether the square radius of gyration vanishes or not in the thermodynamic equilibrium state. The present method does not appear to give the state of zero radius because the entropy force due to random walks is balanced with the attractive force. In the present model, the vanishing of the square

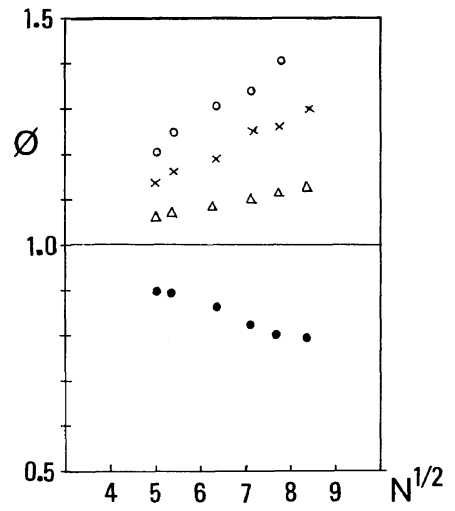

Figure 5. Expansion factor $\Phi$ as a function of $N^{1 / 2}$ for various values of $\kappa: \mathrm{a}(\bigcirc), \kappa=1.2 ; \mathrm{b}(\times), \kappa=0.6 ; \mathrm{c}(\triangle)$, $\kappa=0.2 ; \mathrm{d}(\mathrm{O}), \kappa=-0.2$.

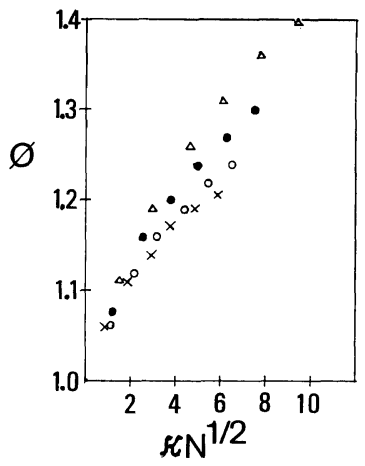

Figure 6. Expansion factor $\Phi$ as a function of $\kappa N^{1 / 2}$. Data taken from Figure 4: a $(\triangle), N=60 ; \mathrm{b}(\bigcirc), N=40$; c (O), $N=30$; d (×), $N=25$.

radius of gyration $\left\langle S^{2}\right\rangle$ may depend on the potential range $d$ in the case of negative $\kappa$. The fact that $\left\langle S^{2}\right\rangle$ and $\left\langle R^{2}\right\rangle$ are independent of the potential range in the repulsive force has been established for large $N$ by a variety of theoretical and experimental studies. Since it is not clear whether this independence holds for pure attractive forces, it is desirable to examine the behavior of $\left\langle S^{2}\right\rangle$ as a function of the range $d$ as well as the strength $\kappa$.

According to the two-parameter theory, the expansion factor $\Phi$ can be expanded in terms of $z$ as

$$
\begin{gathered}
\Phi=\left\langle S^{2}\right\rangle /\left\langle S^{2}\right\rangle_{0} \\
=1+C_{1} z-C_{2} z^{2}+C_{3} z^{3}-\cdots \\
z=\left(3 / 2 \pi a^{2}\right)^{3 / 2} \beta N^{1 / 2}
\end{gathered}
$$

where $\beta$ is the binary cluster integral (for the values 
of $C_{1}, C_{2}$, and $C_{3}$; see Yamakawa ${ }^{27}$ ). This expansion is exact only in the limits $N \rightarrow \infty, \beta \rightarrow 0$, and $\beta N^{1 / 2}=$ finite. Since $\beta$ and $N$ appear in $z$ as a single variable $\beta N^{1 / 2}$, it is important to analyze the behavior of $\Phi$ not only as a function of $\beta$ and $N^{1 / 2}$ but also as a function of $\beta N^{1 / 2} \cdot{ }^{28}$ Note that in the present model $z=f \kappa N^{1 / 2}$, where $f$ is the scale factor and may be equated to unity without loss of generality. Figure 5 and 6 show $\Phi$ as functions of $N^{1 / 2}$ and $\kappa N^{1 / 2}$, respectively. Equations 28 and 29 state that data of $\Phi$ for different pairs of $N$ and $\kappa$ fall on a single curve. Such behavior seems to hold for $\kappa N^{1 / 2}<2$, but not for $\kappa N^{1 / 2}>2$. In order to clarify this point, simulations for very large values of $N$ and small $\kappa$ are required. Probably, this will give significant information, since the present method resorts to unrestricted random walk as in the case of the two-parameter theory.

\section{CONCLUDING REMARKS}

It should be pointed out that the results of the present study are qualitative rather than quantitative because of the insufficient number of samples and unsuitable parameters associated with the potential adopted. Particularly for $d$, the choice of a somewhat smaller value might have been desirable. The validity of the present method will be established by comparing the behavior calculated for the Lennard-Jones 12-6 potential by the present method with that by the dynamic Monte Carlo method, for which many useful data are available. ${ }^{29}$

The purpose of this paper has been to make the first application of the method developed by Tsuda et al. to polymer problems. Neither the concept used nor the method itself is new to the polymer problems. The uniqueness of this method lies in the construction of the zig-zag Wiener path (see eq 21). Up to now, this method has not been used for simulation. However, eq 21 is limited to a random walk which starts from a fixed point and ends up at another fixed point. This greatly facilitates the study of polymer conformations since usual static Monte Carlo method is not valid for such a conformation. This method may also provide considerable insight into the statistical properties of a ring chain in the unperturbed state, because difficulty associated with the interaction potential does not appear in this case.

The present method can also be applied to a chain interacting with a wall through an appropriate onebody potential. Of course, in this case, the ensemble of Wiener paths consists of unrestricted random walks. Equation 21 should be transformed into a different form so as to describe the unrestricted random walk repelled elastically by the wall. This can be achieved if we utilize the fundamental properties of Brownian motion (reflection principle). It is, however, impossible to describe the system placed in a one-body potential undergoing rapid variation within a range comparable to the step length.

The method for constructing Wiener paths is not limited to the use of eq 21. Levy and also Paley and Wiener developed mathematically rigorous theories for constructing these paths. Since eq 21 is not valid for linear chains, we must use their methods for such chains, especially the method of Paley and Wiener, in which the Fourier expansion technique is used.

Finally, it may be noted that studies of the two problems mentioned above are now in progress. The results will be published in the near future, ${ }^{30}$ along with a discussion on the statistical errors associated with simulation, which have not been touched upon in this paper.

Acknowledgments. The authors are grateful to Professor S. Koide for his critical reading of the manuscript and helpful comments. This work was supported in part by a Grant-in-Aid for Scientific Research from the Ministry of Education, Science and Culture of Japan.

\section{REFERENCES}

1. T. Tsuda, K. Ichida, and T. Kiyono, Numerische Mathematik, 10, 110 (1967); T. Tsuda, "Monte Carlo Method and Simulation,” (in Japanese), Baifukan, Tokyo, 1977, p 162.

2. Precise estimation of the path integral by Monte Carlo simulation is found; G. Sher, M. Smith, and M. Baranger, Anal. Phys., 130, 290 (1980).

3. For review on these methods as applied to polymers see; C. Domb, Adv. Chem. Phys., 15, 229 (1969); D. S. McKenzie, Phys. Rep., 27C, 35 (1978).

4. F. T. Wall, S. Windwer, and P. J. Gans, "Monte Carlo Method applied to Configuration of Flexible Polymer Molecules," in Method of Computational Physics, Vol. 1, Academic Press, New York, N. Y., 1963.

5. P. G. de Gennes, Phys. Lett., 38A, 339 (1972). 
6. A. Baumgärtner and K. Binder, J. Chem. Phys., 71, 2541 (1979); A. Baumgärtner, ibid., 72, 871 (1980); A. Baumgärtner, ibid., 73, 2489 (1980).

7. D. Ceperley, M. H. Kalos, and J. L. Lebowithz, Macromolecules, 14, 1472 (1981); I. Webman, J. L. Lebowitz, and M. H. Kalos, ibid., 14, 1495 (1981); I. Webman, J. L. Lebowitz, and M. H. Kalos, Phys. Rev., B21, 5540 (1980); I. Webman, J. L. Lebowitz, and M. H. Kalos, Phys. Rev., A23, 316 (1981).

8. Dynamic Monte Carlo method has been applied to multiple chain systems. See, A. Baumgärtner and K. Binder, J. Chem. Phys., 75, 2994 (1981).

9. P. G. de Gennes, "Scaling Concepts in Polymer Physics," Cornell University Press, Ithaca, N. Y., 1979.

10. See, for example, ref 4.

11. P. G. de Gennes, Macromolecules, 13, 1069 (1980); P. Dejardin and R. Varoqui, J. Chem. Phys., 75, 4115 (1981); A. T. Clark and M. Lal, "The Effect of Polymers on Dispersion Properties," Th. F. Tadros, Ed., Academic Press, New York, 1982, p 169, and references cited therein.

12. S. Chandrasekhar, Rev. Mod. Phys., 15, 1 (1943).

13. R. P. Feynman and A. R. Hibbs, "Quantum Mechanics and Path Integrals," McGraw-Hill, New York, N. Y., 1965; R. P. Feynman, "Statistical Mechanics," Benjamin, Massachusetts, 1972.

14. K. F. Freed, Adv. Chem. Phys., 22, 1 (1972).

15. P. Levy, "Processus Stochastiques et Mouvement
Brownien," Gauthier-Villars, 1965.

16. R. E. A. C. Paley and N. Wiener, "Fourier Transforms in the Complex Domain," American Mathematical Society Colloquim Pub. XIX, 1934.

17. T. Hida, "Brownian Motion," in Japanese, Iwanami, Tokyo, 1975.

18. A. Baumgärtner and K. Binder, J. Chem. Phys., 71, 2541 (1979).

19. F. L. McCrackin, J. Mazur, and C. M. Guttman, Macromolecules, 6, 859 (1973).

20. N. C. Smith and R. J. Fleming, J. Phys. A, 8, 938 (1975).

21. W. Bruns, J. Phys. A, 10, 1963 (1977).

22. S. F. Edwards, Proc. Phys. Soc., 85, 613 (1965).

23. J. des Cloizeaux, J. Phys. (Paris), 42, 635 (1981).

24. T. Minato, K. Ideura, and A. Hatano, Polym. J., 14, 579 (1982).

25. See, for example, ref 7,19 , and 20.

26. C. Domb, Polymer, 15, 259 (1974); S. F. Edwards, in "Critical Phenomena," Natural Bureau of Standards Miscell. Pub., Washington, D. C., 1966.

27. H. Yamakawa, "Modern Theory of Polymer Solutions," Harper and Row, New York, N. Y., 1971.

28. C. Domb, A. J. Barrett and M. Lax, J. Phys. A, 6, L82 (1973).

29. See, for example, ref $6,7,8$, and 18 .

30. T. Minato and A. Hatano, in preparation. 